\title{
Numerical simulation of a modified multiple fractured well model in tight gas reservoirs
}

\author{
Chunyuan $\mathrm{Xu}^{1, \mathrm{a}^{*}}$, Detang $\mathrm{Lu}^{1, \mathrm{~b}}$ \\ 1 Oil and Gas Research Centre, University of Science and Technology of China, Hefei 230026, \\ China
}

a xcyuan@mail.ustc.edu.cn, b dtlu@ustc.edu.cn

Keywords: discrete fracture, multiple fractures, equivalent fracture, closure, productivity.

Abstract. Most fracture patterns near the well region are more fickle in tight gas field than that in literature. In this paper, a modified discrete fractured well model is presented to account for dynamic characteristics of a vertical well with multiple asymmetrical fractures (MAF). Fracture closure effect is also coupled in the revised numerical model, leading to a more rapidly loss of gas production. The productivity of a vertical well with MAF is investigated by comparing to a fractured well with one single equivalent fracture (EF). Furthermore, the effect of fracture conductivity on cumulative production is discussed. Results show that EF representation can cause up to 45.4 percent less production than MAF. Fracture conductivity is proved to be closely related to cumulative production.

\section{Introduction}

The oil and gas field has long recognized the uncertainty of fractured well morphology. With the wide-spread development of tight gas reservoirs in which fluid flow is mostly through high conductive fractures, it has become increasingly critical to establish a more accurate fractured well model for field development guidance. For vertical wells with SRV treatments, recent advances in fracture diagnostic techniques prove a vertical well with MAF to be more realistic (Fisher et al., 2005, Craig \& Blasingame, 2006). Existing models are not adequate for predicting performance of wells in this kind, especially when hydraulic fractures tend to shrink during the production period in tight gas reservoirs.

There has been some works on multiple fractures intersecting at one cross-point that locates in the well position. Nearly all of them are analytical methods because of their relatively high efficiency in calculations compared to the numerical methods. For vertical fractured wells, where the fractures are non-single and arbitrarily oriented, Craig \& Blasingame (2006) derived an analytical solution to study the pressure transient behavior. They presented the cruciform situation in particular to show the details of the solution. Afterward, Restrepo \& Tiab (2009) established a new analytical model considering wellbore storage and skin effect for a similar fracture pattern. Tiab's direct synthesis Technique (TDS) was used to obtain parameters of the reservoir and fractures. The effect of fracture asymmetry was also recognized and discussed in some literatures (Wang et al., 2013, Tiab et al., 2010). On the basis of previous work, Chen (2014) and Luo (2014) analyzed the productivity and transient pressure behaviors of vertical wells with MAF respectively. However, none of these approaches has considered the fracture closure effect, which is a common phenomenon during hydraulic fracturing operations. Some researchers thought stress alteration is the main reason for fracture compression (Moinfar, 2013, Aybar, 2014).

The numerical discrete fracture model (DFM), though time consuming, provides an effective way to model flow in complex fracture network by using a lower dimensional representation of fractures in the grid domain. In the past decade, there has been increasing attention on tight gas simulation by DFM (Gong B, 2011, Zidane \& Firoozabadi, 2014). In general, DFM is more adaptive to various mechanisms when compared to analytical models and hence is adopted in this paper.

We extend the idea of reducing dimension from fractures to wells and propose a modified multiple fractured well model in consideration of fracture closure effect. The productivity analysis of the new well model is the main focus of the following sections. 


\section{Physical Model}

Fig. 1 is a schematic of flow in the physics domain and grid domain. The vertical well and fractures are represented by $0 \mathrm{D}$ entity and $1 \mathrm{D}$ entities respectively in a lower dimensional form. Physical model assumptions are made as follows:

I The reservoir contains a single phase gas with variable density and viscosity.

I Isothermal flow occurs in the homogeneous and isopachous reservoir.

I Darcy's law, dynamic slippage and adsorption/desorption is considered in the matrix while flow in the fractures obeys Darcy's law.

I The layer is penetrated by finite-conductivity fractures, which has changing apertures.

I Wellbore storage and skin effect are neglected in this model.
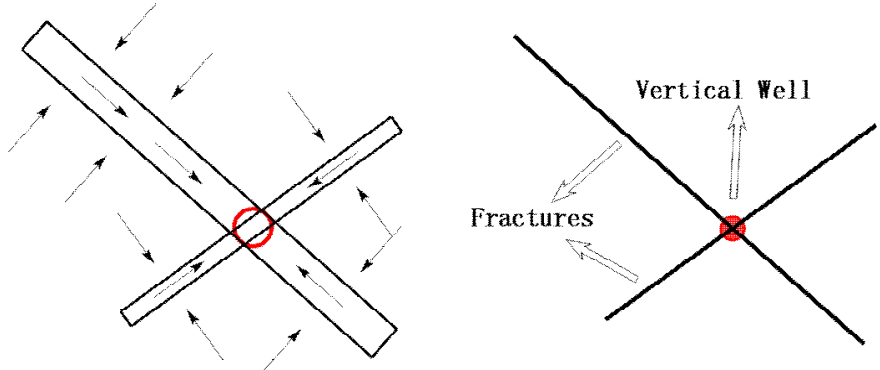

Fig. 1. Left side: flow near a vertical well with two AF. Right side: discrete representation of the fractured well.

\section{Mathematical Model}

\section{Matrix equation}

The equation describing matrix flow is stated as:

$$
\nabla \bullet\left(\frac{\rho_{m} k_{m}}{\mu_{m}} \nabla p_{m}\right)=\frac{\partial}{\partial t}\left(\phi_{m} \rho_{m}+m_{a d s}\right)+q_{m}
$$

Where the subscript $m$ represents the matrix medium. $m_{a d s}$ is the adsorption(desorption) term and $q_{m}$ is the source(sink) term. $\mu$ and $\rho$ are viscosity and density of gas respectively.

A modified apparent permeability is introduced to account for dynamic slippage effect (Beskok, 1999):

$$
k_{m}=k_{m, \infty}\left[1+\alpha\left(K_{n}\right) K_{n}\right]\left[1+\frac{4 K_{n}}{1-K_{n}}\right]
$$

Adsorption of the gas is represented by the Langmuir isotherm:

\section{Fracture Equation}

$$
V=\frac{V_{L} p_{m}}{p_{m}+p_{L}}
$$

Fractures are high permeable media in tight gas reservoirs. Dynamic slippage effect and adsorption/desorption can be neglected because of high pore throat radius and low organic content. Therefore, the continuity equation satisfies Darcy's law:

$$
\nabla \bullet\left(\frac{\rho_{f} k_{f}}{\mu_{f}} \nabla p_{f}\right)=\frac{\partial}{\partial t}\left(\phi_{f} \rho_{f}\right)+q_{m}
$$

Where the subscript $f$ represents the fracture medium.

Fractures compression results from the increase of effective stress over time. According to Biot's elastic equation, effective stress is computed by subtracting the pore pressure from constant total stress:

$$
\sigma=S-p_{f}
$$


As the pore pressure decreases with continuous production from the reservoir, an increasing effective stress is applied on the fractures and hence changes fracture aperture (Bandis et al., 1983):

$$
w_{i}=w_{i 0}-\frac{w_{\text {imax }} \sigma}{K_{n i} w_{\text {imax }}+\sigma}
$$

Where $w_{\max }$ is the maximum fracture closure. $\sigma$ is the effective stress. $K_{n i}$ is the initial stiffness of the rock.

As the rock type varies, the variations of fracture aperture are not the same. Fig 2 depicts the changes of fracture aperture with stress under different stiffness and equal maximum closure (the initial fracture aperture is set to unity).

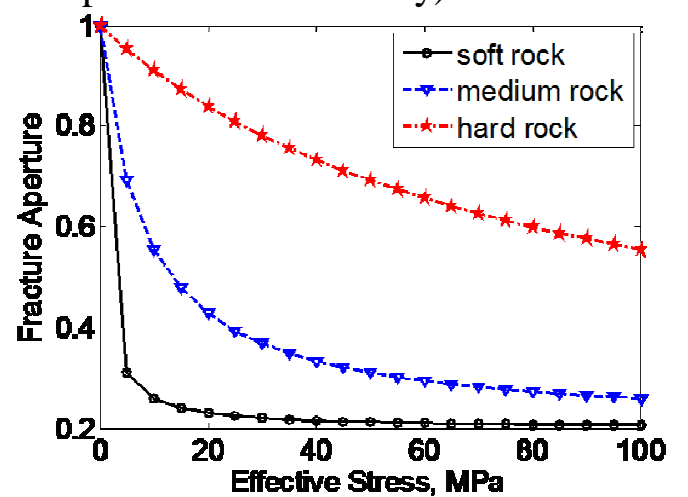

Fig. 2. Evolution of fracture aperture with effective stress.

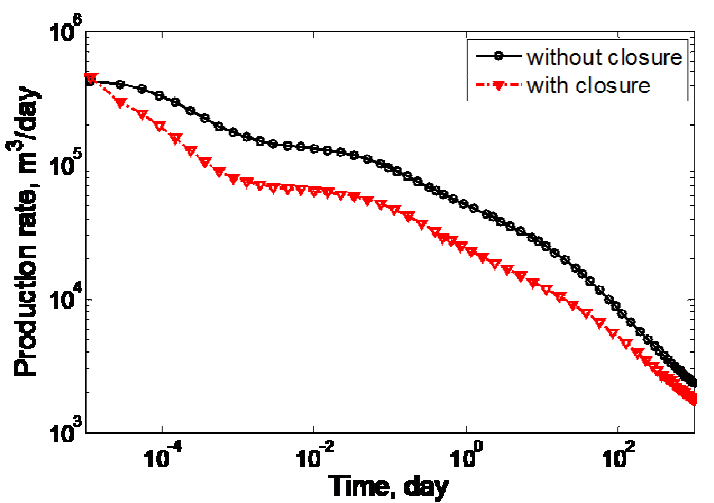

Fig. 3. Log-Log plot of gas production rate over time.

Changes of fracture porosity and fracture permeability based on stress are also included in this model. However, details of the correction are omitted for simplicity.

Well index

The most important parameter that links flow from fractures to the wellbore is Well Index (WI). WI is traditionally constant while changing in this model for a particular well:

$$
W I_{i}=\frac{k_{f} w_{i} h}{r_{i}}
$$

Noted that different fractures do not share the same WI .

\section{Productivity Analysis}

Productivity of the new well model was examined through three aspects: fracture closure effect, equivalent representation and fracture conductivity. A vertical well with ten wings was designed for the analysis of the first aspect. Fracture aperture and permeability were set identical for the equivalence hypothesis. To investigate the influence of fracture parameters, fractures with low conductivity $\left(\mathrm{F}_{\mathrm{CD}}<\right.$ 200), which reflect finite conductive features of the geologic entities, was taken into consideration.

\section{Fracture closure effect}

For the analysis of fracture closure effect, Log-Log plot of gas production rate over time is shown in Fig 3. It is observed that, at the beginning of the production, the gas rate with closure effect decreases faster than that without closure effect. Snapshots of pressure distribution after 1 day and 1000 days are shown in Fig 4 and Fig 5, respectively. Fig 4 shows that pressure profile with and without considering fracture closure effect are similar after 1 day. However, a lower pressure drop, as can be seen in Fig 5, exhibits after 1000 days in consideration of fracture closure effect, which manifests that fracture closure effect has an apparent influence on long term production behavior of the vertical well with MAF. 

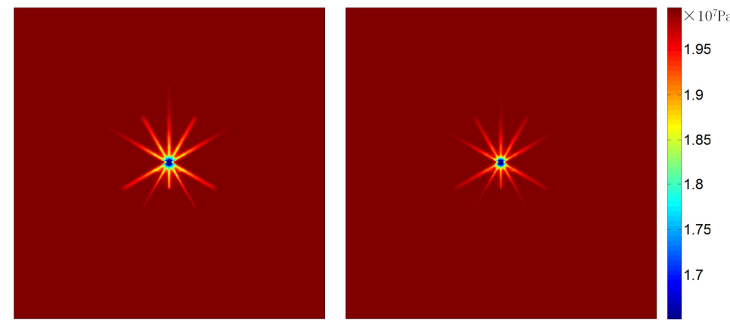

Fig. 4. Snapshots of pressure distribution after 1 day. Left side: without fracture closure.

Right side: with fracture closure.
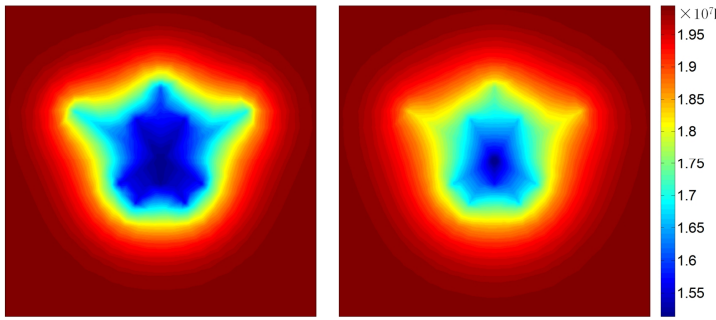

Fig. 5. Snapshots of pressure distribution after 1000 days. Left side: without fracture closure. Right side: with fracture closure.

It is common practice to represent MF with EF in tight gas simulation. Fig 6 shows the history of cumulative gas production in consideration of fracture closure effect. The cumulative production of vertical well with MF can be up to $45.4 \%$ higher than the well with EF. It is concluded that the equivalent representation tends to underestimate well productivity.

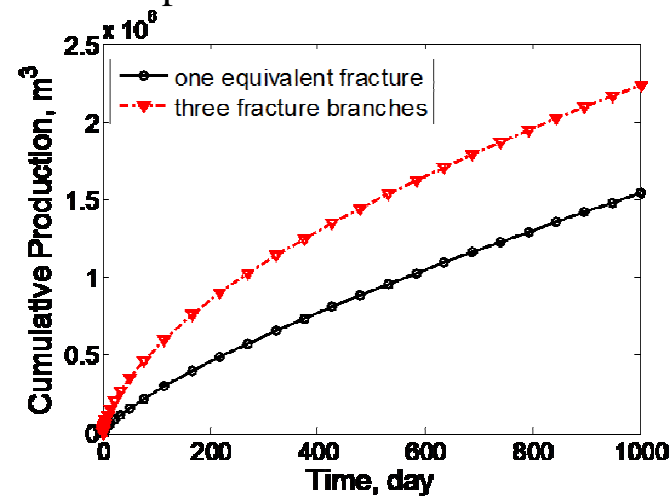

Fig. 6. History of cumulative gas

production over 1000 days with fracture closure effect.

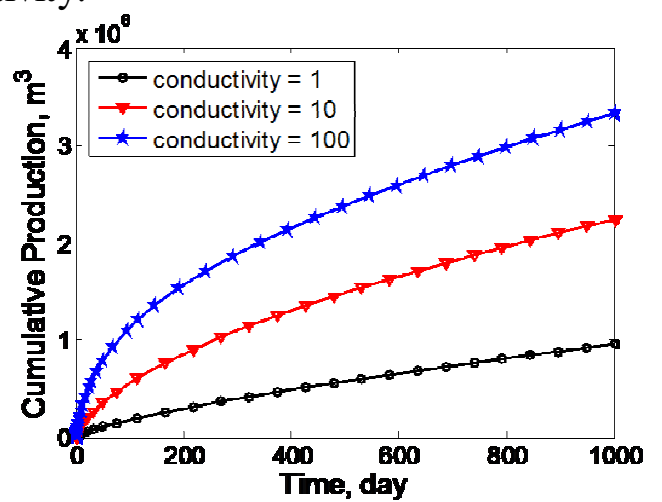

Fig. 7. History of cumulative gas production under different fracture conductivity.

\section{Fracture conductivity}

Fig 7 presents the influence of fracture conductivity on well productivity. It is obvious that well productivity increases with increasing fracture conductivity.

\section{Conclusion}

A modified numerical solution coupled fracture closure effect for vertical wells with MAF is presented. Following conclusions are obtained:

I Fracture closure effect has an apparent influence on long term production behavior of the vertical well with MAF.

I The productivity of wells with MF is higher when compared to those with EF.

I Well productivity is strongly affected by fracture conductivity.

\section{References}

[1] Aybar U, Eshkalak M O, Sepehrnoori K, et al. 2014. The ef-fect of natural fracture's closure on long-term gas pro-duction from unconventional resources[J]. Journal of Natural Gas Science and Engineering 21: 1205-1213.

[2] Bandis S C, Lumsden A C, Barton N R. 1983. Fundamentals of rock joint deformation. International Journal of Rock Mechanics and Mining Sciences \& Geomechanics Ab-stracts 20(6): 249-268.

[3] Beskok A, Karniadakis G E. 1999. Report: a model for flows in channels, pipes, and ducts at micro and nano scales[J]. Microscale Thermophysical Engineering 3(1): 43-77. 
[4] Craig, D. P., \& Blasingame, T. A. 2006. Constant-Rate Drawdown Solutions Derived for Multiple Arbitrarily-Oriented Uniform-Flux, Infinite-Conductivity, or Finite-Conductivity Fractures in an Infinite-Slab Reservoir. SPE Gas Technology Symposium. Society of Petroleum Engineers.

[5] Fisher M K, Wright C A, Davidson B M, et al. 2005. Inte-grating fracture mapping technologies to improve stimu-lations in the Barnett Shale[J]. SPE Production \& Facili-ties 20(02): 85-93.

[6] Gong B, Qin G, Douglas C, et al. 2011. Detailed Modeling of the Complex Fracture Network and Near-well Effects of Shale Gas Reservoirs[C]. SPE Middle East Unconven-tional Gas Conference and Exhibition. Society of Petro-leum Engineers.

[7] Moinfar A, Sepehrnoori K, Johns R T, et al. 2013. Coupled geomechanics and flow simulation for an embedded dis-crete fracture model[C]. SPE Reservoir Simulation Sym-posium. Society of Petroleum Engineers.

[8] Restrepo D P, Tiab D. 2009. Multiple Fractures Transient Response[J]. Latin American and Caribbean Petroleum Engineering Conference. Society of Petroleum Engi-neers.

[9] Tiab D, Lu J, Nguyen H, et al. 2010. Evaluation of Fracture Asymmetry of Finite-Conductivity Fractured Wells[J]. Journal of Energy Resources Technology 132(1): 012901.

[10] Wang L, Wang X, Li J, et al. 2013. Simulation of pressure transient behavior for asymmetrically finite-conductivity fractured wells in coal reservoirs[J]. Transport in porous media 97(3): 353-372.

[11] Wanjing L, Changfu T. 2014. Pressure-Transient Analysis of Multiwing Fractures Connected to a Vertical Well-bore[R]. SPE Journal.

[12] Zhiming C, Xinwei L, Chenghui H, et al. 2014. Productivity estimations for vertically fractured wells with asymmet-rical multiple fractures[J]. Journal of Natural Gas Sci-ence and Engineering 21: 1048-1060.

[13] Zidane A, Firoozabadi A. 2014. An efficient numerical model for multicomponent compressible flow in frac-tured porous media[J]. Advances in Water Resources 74: 127-147. 